\title{
Motor and cognitive factors in the modification of a reflex
}

\author{
MICHELLE E. COHEN, JACQUELYN CRANNEY, and HOWARD S. HOFFMAN \\ Bryn Mawr College, Bryn Mawr, Pennsylvania
}

\begin{abstract}
By closing a hand-held switch, subjects caused a miniature solenoid to deliver a tap to their own foreheads. (1) The amplitude of elicited eyeblinks was reduced when the delay between switch closure and tap was 50 msec or less. (2) Subjects who expected that switch closure would produce an immediate tap exhibited smaller blinks to such taps than did subjects who expected switch closure to produce a delayed tap. (3) The inhibitory effects of a reflex-modifying tone prior to tap were the same when taps were self-presented as when they were presented by the experimenter. (4) When blinks could be elicited by either a tap or a loud noise, the smallest responses occurred when subjects knew which to expect. (5) On a given trial, the inhibition afforded by the particular stimulus, motor, and cognitive factors operating at the time tended to add in an algebraic fashion.
\end{abstract}

When a mild, sensory event, such as a weak tone or a dim light flash, precedes a reflex-eliciting signal by an appropriate interval, the reaction is often reduced. This reflex-modification effect has broad generality. It occurs in amphibians (Yerkes, 1905), birds (Stitt, Hoffman, Marsh, \& Schwartz, 1976), and mammals, including man (Hoffman \& Ison, 1980). Moreover, it occurs with a variety of reflexes and with a variety of reflex-eliciting and reflex-modifying signals. When, for example, a barely audible tone is presented $100 \mathrm{msec}$ before delivery of an abrupt tap to a subject's glabella (the flattened region of skin between the eyebrows), the eyeblink that the tap ordinarily elicits either fails to occur or occurs with greatly diminished amplitude. The effect does not depend upon learning. It occurs the first time the tone precedes the tap. Nor does the effect depend upon the subject's motor and/or cognitive activity at the time the reflex is elicited. Reflex modification has been assessed while subjects were sleeping (Silverstein \& Graham, 1979), while they were reading (Dykman \& Ison, 1979), and while they were watching a slide show (Hoffman, Cohen, \& Stitt, 1981).

This is not to say that motor and/or cognitive processes have no role in the reflex-modification effect. On the contrary, one can easily observe a form of reflex modification which is at least as powerful as that exerted by a properly presented exteroceptive stimulus but which depends critically upon a subject's motor and/or cognitive activities. To do so,

This research was supported by National Institutes of Health Grant HD 10511. Requests for reprints should be sent to Howard S. Hoffman, Department of Psychology, Bryn Mawr College, Bryn Mawr, Pennsylvania 19010. We wish to thank Laura H. Daruns for suggesting the research strategy employed in Experiment 4. one need only to try to elicit an eyeblink in oneself by tapping one's own glabella with a forefinger. If the reaction (or more likely the lack of one) is compared with the vigorous blink that occurs when a friend delivers the tap, it becomes clear that the act of selfpresenting a stimulus can somehow exert powerful inhibitory control over the reaction that is elicited. The research reported here was designed to examine the motor and cognitive factors that might be responsible for this effect.

Previous investigators have noted that if strong aversive stimuli are either self-presented or forewarned, they are often reported as seeming weaker than when the same stimuli are presented unexpectedly (Bjorkstrand, 1973; Grings, 1960; Haggard, 1943; Maltzman \& Wolff, 1970; Staub, Tursky, \& Schwartz, 1971). Lykken and Tellegen (1974) have proposed that this form of "negative perception" implies that subjects can selectively tune the appropriate afferent system in anticipation of a stimulus and that when the stimulus is aversive the effect is to attenuate its perceived impact. Furedy and Klajner (1974), however, have challenged this proposal on methodological grounds. They noted that strong aversive stimuli (such as electrical shock) would be expected to induce large increases in arousal, and they questioned whether or not self-presentation and/or forewarning might not produce its major effects by attenuating arousal rather than by directly influencing the afferent input from the aversive stimulus.

In the research reported here, we hoped to, in part, avoid the problem noted by Furedy and Klajner by employing relatively innocuous stimuli. It seemed clear that the taps to the glabella to be used here would be much less likely to induce arousal than the electrical shocks that were used in most prior studies. 
Moreover, rather than relying on reports of perceived stimulus intensity, the present research would directly measure the amplitude of the eyeblinks that the taps elicited. By doing so, we hoped to avoid the potential problems of interpretation that arise when reports of perceived intensity constitute the basic datum.

\section{EXPERIMENT 1}

Experiment 1 was designed to assess the temporal course of the self-presentation effect. In it, student volunteers were instructed to close a hand-held switch so as to initiate an eyeblink-eliciting tap to their own glabellas. On some trials, the tap occurred immediately upon the switch closure, but on others, it was delayed by an amount that varied from trial to trial.

\section{Method}

Subjects. Twelve (four female, eight male) graduate and undergraduate students from Bryn Mawr College participated in this study.

Appantus. The devices for delivering a controlled tap to the glabella and for monitoring the reflexive eyeblink that it elicited have been described in detail elsewhere (Marsh \& Hoffman, 1981; Marsh, Hoffman, \& Stitt, 1979). The tap unit consisted of a miniature solenoid with a small (5-mm-diam) ball of silicone rubber securely fastened to its plunger. When the solenoid was activated, the ball was thrust forward with an impulse (in newtons/second) that, as measured with a ballistic pendulum, was equal to .95 times the applied voltage.

The eyeblink monitor consisted of a miniature optical device $(1 \times .5 \times .5 \mathrm{~cm})$ that contained a source of infrared light and a sensitive phototransistor pickup. The output of this pickup was amplified and filtered electronically before being sent to a storage oscilloscope.

The research was conducted in an IAC double-wall soundtreated chamber with an ambient noise level below $25 \mathrm{dBA}$ (unless otherwise noted, all intensities are re .0002 dynes $/ \mathrm{cm}^{2}$ ). This chamber was suitably furnished and lighted. It contained a small translucent screen for displaying colored slides projected from outside the chamber. It also contained a closed-circuit television camera and an intercom to permit monitoring of and continuous communication with the subject.

Procedure. After a given subject had been apprised of the nature of the research and had agreed to participate, he or she was seated in the IAC room and fitted with the headband holding the tap unit and the eyeblink monitoring device. The subject was then informed that throughout the experiment colored slides of nature subjects as well as artworks would be projected onto the screen and that, while the primary purpose of the slides was to prevent boredom, each slide change would signal that the button of a plunger-type switch held in the subject's preferred hand should be pressed after a wait of about $10 \mathrm{sec}$. He or she was also told that each switch closure would be likely to cause a tap to occur, but that the interval between the switch closure and the tap would vary from trial to trial.

Taps to the glabella were produced by delivering a 50 -msec, $16-$ $V$ dc pulse to the tap solenoid. On a given trial, the switch closure was either without effect or it yielded a tap with an onset delay of $0,50,500$, or $5,000 \mathrm{msec}$. Trials occurred at intervals of $20 \pm 5 \mathrm{sec}$ and were programmed so that each block of five trials contained a different randomization of the five conditions. In a session that lasted approximately $\mathbf{4 0} \mathrm{min}$, each subject received 10 trials of each of the four delay tap conditions as well as $\mathbf{1 0}$ trials of the no-tap condition. This arrangement was designed to enable us to assess the time course of the inhibitory effect of self-presentation and also determine if the self-presentation act (switch closure) might itself become a source of conditioned eyeblinks. On tap trials, amplitude of the eyeblink was measured during the $100 \mathrm{msec}$ that began with the onset of the tap. For trials in which switch closure did not yield a tap, potential conditioned blinks were measured during the 100 msec that began with the onset of the switch closure.

\section{Results}

Since no subject ever exhibited a measurable eyeblink to switch closure without a tap, it is concluded that these procedures did not generate some form of conditioned eyeblinks that might have interfered with the eyeblinks elicited by the taps.

Figure 1 shows the mean amplitude of these tapelicited eyeblinks (averaged across subjects) at each temporal delay. The shorter the delay between the switch closure and the tap, the smaller elicited eyeblink. This is the basic self-presentation effect, and, as seen in Figure 1, it largely disappeared when the delay between switch closure and tap was as long as $500 \mathrm{msec}$. A repeated-measures analysis of variance was carried out to assess the statistical reliability of this finding. It yielded an $F(4,44)=40.85, p<.01$. A subsequent Newman-Keuls analysis indicated that, while both the 0 - and $50-\mathrm{msec}$-delay conditions were significantly different from the 500 - and 5,000msec-delay conditions $(p<.05)$, neither the difference between the 0 - and $50-\mathrm{msec}$ delay nor the difference between the 500- and 5,000-msec delay was statistically significant.

\section{Discussion}

As revealed here, the inhibition afforded by selfpresentation of a reflex-eliciting stimulus diminished relatively rapidly as the interval between the act of self-presentation (e.g., the switch closure) and the occurrence of the tap increased. These results agree with the findings of Maltzman and Wolff (1970);

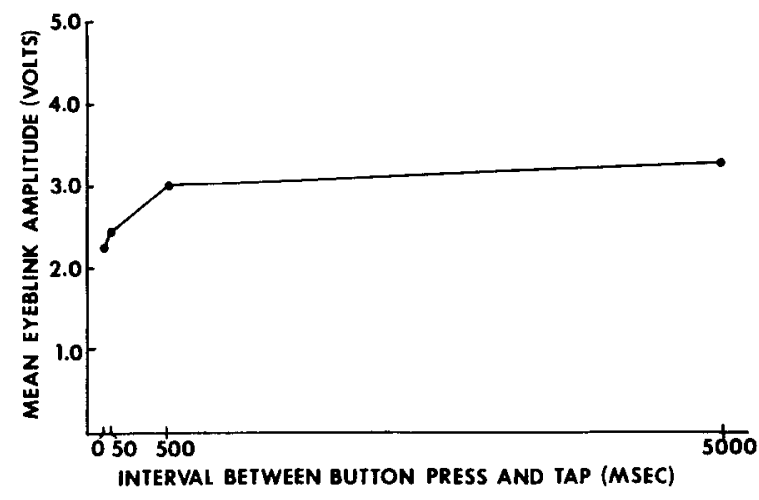

Figure 1. Mean amplitude of ejeblink elidted by a self-presented tap to the glabella (the fint patch of alin between the ejebrows) as $a$ function of the interval between the self-presentation reaponse (a buttonpreas) and tap delivery. 
individuals react less to an immediate stimulus than to one delayed by an unpredictable amount of time. For present purposes, it is of interest that this finding can be interpreted in any of a variety of ways. For example, it may mean that the overt self-presentation response (or the neural command that initiates it) generates an inhibitory process that serves to reduce the amplitude of a subsequently elicited reflex. If it does, then the present findings imply that this inhibitory process is relatively short lived, since, as seen in Figure 1, the inhibitory effect engendered by selfpresentation persisted for less than $500 \mathrm{msec}$ after the overt response.

Another possibility is that, rather than depending upon the occurrence of a motor response (or the neural command that initiates it), the inhibition engendered by self-presentation depends upon the subject's set or expectation at the moment of stimulus presentation. If this is the case, our results imply that the set or expectation associated with selfpresentation persists for more than $50 \mathrm{msec}$ but less than $500 \mathrm{msec}$ after the overt response.

Perhaps a more likely possibility is that the motor response (and/or the neural command that initiates it) and the subject's expectation at the moment the response is made jointly contribute to the reflex inhibition engendered by self-presentation. If so, our results again imply that the inhibition engendered by these processes persists for somewhere between 50 and $500 \mathrm{msec}$ after the overt self-presentation response has been initiated.

In analyzing the reflex inhibition afforded by selfpresentation, Sanes (1979) has concluded that this inhibition is probably initiated prior to the occurrence of the motor response. The data obtained here are consistent with this proposition. Previous studies in this and other laboratories reveal that when a weak tone precedes a more intense startle-eliciting signal, the reaction to the intense signal is maximally inhibited when the lead interval approximates $100 \mathrm{msec}$ and little, if any, inhibition occurs when the lead interval is less than 10 msec (Graham \& Murray, 1977; Hoffman \& Ison, 1980; Krauter, Leonard, \& Ison, 1973). In the case of self-presentation, however, inhibition is maximal when the interval between switch closure and the blink-eliciting signal is $0 \mathrm{msec}$. A reasonable way to account for this discrepancy is to assume that the inhibition afforded by self-presentation is mediated by some event that occurs just prior to the switch closure. The most obvious possibility is that this event consists of the neural command that initiates this behavior.

\section{EXPERIMENT 2}

While the results of Experiment 1 are consistent with the hypothesis that a subject's set or expecta- tion may act with some aspect of the motor (or motor command) system to jointly inhibit an elicited reflex, the design of Experiment 1 does not permit one to, in fact, separate these two possible sources of reflex inhibition. Experiment 2 was designed to accomplish this goal. In Experiment 2, subjects were again required to initiate their own taps by closing a switch. Now, however, the taps were either immediate or delayed by $5,000 \mathrm{msec}$, and the probabilities of a delayed versus immediate tap were such that subjects in one group would expect an immediate tap on most trials, whereas subjects in a second group would expect a delayed tap on most trials. With this arrangement, the effects of the differing expectations should be revealed in the comparisons between the two groups on trials when taps were immediate as well as on trials when taps were delayed.

\section{Method}

Subjects. Twenty-four (19 female, 5 male) undergraduate and graduate students from Bryn Mawr College volunteered to participate in this study. The subjects were placed randomly into one of two groups of $n=12$ each.

Apparatus. The equipment used for this study was the same as that used in Experiment 1.

Procedure. After a subject had been apprised of the nature of the research and had agreed to participate, he or she was seated in the IAC room and fitted with the headband holding the tap device and the eyeblink monitoring device. As in Experiment 1, the subjects were informed that slides of nature and/or art subjects would be projected onto the screen in front of them and that they were to present a tap to themselves by pressing the button of a hand-held plunger-type switch approximately $10 \mathrm{sec}$ after each slide change. They were also told that sometimes the tap would occur immediately and sometimes the tap would be delayed by a few seconds.

For subjects in Group A, the probability of an immediate tap was .75 and the probability of a delayed tap was .25 . These subjects received 30 trials in which the tap immediately followed the switch closure and 10 trials in which the tap was delayed by 5,000 msec. For subjects in the second group (Group B), these probabilities were reversed. These subjects received 30 trials on which the tap was delayed by $5,000 \mathrm{msec}$ and 10 trials in which the tap immediately followed a switch closure. For all subjects, trials occurred in a random order, at intervals of $20 \pm 5 \mathrm{sec}$, in a single session that lasted approximately $30 \mathrm{~min}$.

\section{Results}

Figure 2 shows the mean amplitude (averaged across subjects within each group) of eyeblinks elicited by immediate versus delayed taps. Overall, immediate taps resulted in smaller reflexive blinks than taps that were delayed, and regardless of whether taps were immediate or delayed the condition with the higher probability yielded the smallest responses. A two-factor mixed-design analysis of variance yielded a significant overall effect of delay $[F(1,22)=$ 49.78, $p<.01$ ] and a significant group $\times$ delay interaction $[\mathrm{F}(1,22)=18.79, \mathrm{p}<.01]$. Results of subsequent analyses of simple main effects yielded a significant difference between Groups $A$ and $B$ for the immediate and the delayed tap conditions [immediate, $F(1,44)=4.44, p<.05$; delayed, $F(1,44)=4.15$, 


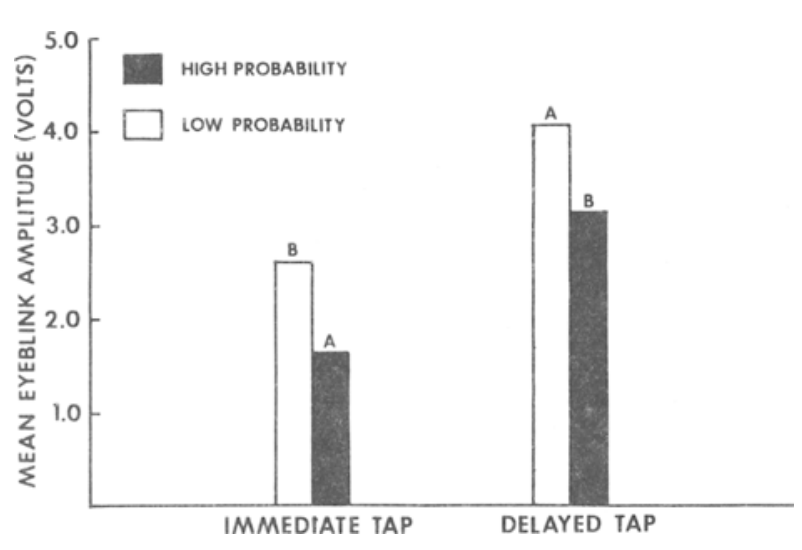

Figure 2. Mean amplitude of eyeblink elleited by a self-presented tap to the glibella. For subjects in Group A (black bar in immediate tap condition, white bar in delayed tap condition), taps were delayed by 5,000 msec on three of four randomly selected trials and taps occurred Immediately on the rest of the trials. For subjects in Group B (white bar in immediate tap condition, black bar in delayed tap condition), taps occurred immediately on three of four trials and tapa were delayed by 5,000 msec on the rest.

$\mathrm{p}<.05]$. This analysis also yielded a significant effect of delay for subjects in Group $A[F(1,22)=$ $65.91, \mathrm{p}<.011$. Within Group B, however, the immediate versus delayed conditions did not result in a significant difference.

\section{Discussion}

In their overall configuration and in their details, the several features of these data point to the conclusion that the amplitude of an elicited eyeblink is reduced when the eliciting stimulus is presented at the time that the subject expects it to occur. These data also provide evidence that the effect does not depend solely on inhibitory processes that are mediated by the occurrence of the motor response (or the neural command that initiates it). To see why, compare the reactions to the immediate tap for subjects in Group A with the reactions to the immediate tap for subjects in Group B. For subjects in both groups, the same motor response preceded each tap by the same interval $(0 \mathrm{msec})$, yet, as shown in Figure 2 and confirmed by the statistical analysis, subjects in Group A exhibited reliably smaller blinks than subjects in Group B. Apparently, in Group A, for which three of four taps were immediate, subjects tended to expect an immediate tap, whereas in Group B, for which only one of four taps was immediate, subjects tended to expect that the tap would be delayed.

As also seen in Figure 2, subjects in Group B gave smaller blinks to delayed taps than did subjects in Group A. This finding is consistent with the fact that for subjects in Group B three of four taps were delayed, whereas for subjects in Group A only one of four taps was delayed. Apparently, subjects in Group B expected delayed taps, and hence their reactions to them were smaller than those of subjects in
Group A, who had expected most taps to be immediate.

Overall, the results of Experiment 2 imply that when exposed to events that have different probabilities, subjects exhibit appropriate expectations and that when stimulus presentation is in accord with expectation, the amplitude of the elicited reflex is reduced. These results are also consistent with the earlier suggestion that the reflex inhibition afforded by self-presentation entails an inhibitory process engendered most likely by the neural command that initiates motor aspects of the self-presentation response. As seen in Figure 2 and confirmed by the statistical analysis, overall, responses to the immediate taps were much smaller than responses to the delayed taps, regardless of the probabilities associated with those taps.

Finally, as seen in Figure 2, the effect of high versus low probability was virtually the same for immediate as for delayed taps. The difference between the amplitudes for the immediate tap conditions equaled the difference in amplitudes for the delayed conditions. In both cases, the expected (high probability) condition yielded the smallest reflexes. This implies that the inhibitory processes engendered by the motor (or motor command) aspects of the selfpresentation response and those engendered by the expectation or set that may accompany this event, make largely independent contributions to the total amount of inhibition generated. Sternberg (1969) discusses a similar finding of the additive effects of expectations in reaction time experiments.

\section{EXPERIMENT 3}

The results of Experiments 1 and 2 suggest that self-presentation of a reflex-eliciting stimulus engages two apparently independent inhibitory processes, both of which serve to reduce the amplitude of the elicited reflex. One of these consists of the motor and/or neural command components of the self-presentation act, and the other consists of the cognitive expectation that accompanies them. As noted in the introduction to this paper, another way to inhibit a reflex is to arrange that the reflex-eliciting stimulus is preceded, at an appropriate interval, by an exteroceptive reflex-modifying signal, such as a mild tone. Experiment 3 was designed to determine how this form of inhibition (e.g., the inhibition mediated by a reflex-modifying tone) might combine with the inhibition mediated by self-presentation (and the expectations ordinarily associated with it).

\footnotetext{
Method

Subjects. Thirteen (six female, seven male) graduate and undergraduate students volunteered to participate in this study.

Apparatus. The equipment used for this study was the same as that used in Experiment 1. The tones that served as reflexmodifying acoustic signals were generated, shaped, amplified, and
} 
timed by Coulbourn modules and were delivered through TDH39 earphones fitied with MX-41/AR cushions. The earphones and test tones were calibrated with a General Radio precision soundlevel meter (Model 1561-A) fitted with a P-7 microphone and an ANSI-type coupler.

Procedure. Each subject was seated in the IAC room and fitted with earphones and the headband holding the tap device and the eyeblink-monitoring device. The subjects were informed that they would be observing a series of colored slides of nature and/or art subjects and that approximately $10 \mathrm{sec}$ after each slide change either they would be told to present a tap to themselves by pressing the button of a hand-held plunger-type switch or tap presentation would be initiated by the experimenter, who would be seated outside the chamber.

During the session, tap presentations occurred at intervals of $20 \pm 5 \mathrm{sec}$ and were arranged so that on $50 \%$ of the trials, the tap would be self-presented and on the rest it would be presented by the experimenter. As in Experiments 1 and 2, each tap was produced by delivery of a 50 -msec, 16-V dc pulse to the tap solenoid, but now, tap, whether self-presented or presented by the experimenter, always occurred with a delay of $150 \mathrm{msec}$.

On half of the trials, the tap was preceded by a $50-\mathrm{msec}, 1-\mathrm{kHz}$, 70-dB SPL tone (rise-fall time $=5 \mathrm{msec}$ ) that began when the trial was initiated. On such trials, switch closure initiated a tone, followed 150 msec later by a tap. On the rest of the trials, switch closure also initiated a tap with a delay of $150 \mathrm{msec}$, but no tone was presented.

Each subject received five of each of the four kinds of trials. For seven of the subjects, the tone was always presented in the right ear. For the other six subjects, the tone was always presented in the left ear. During testing, trials were arranged so that each kind of trial appeared once in each block of four trials in an order that varied from trial block to trial block.

\section{Results and Discussion}

Figure 3 shows the mean amplitude, averaged across subjects, of reflexive eyeblinks elicited when trials were self- versus experimenter-presented and when taps were presented alone or preceded by a reflex-inhibiting signal (e.g., a tone). A two-way repeated-measures analysis of variance yielded significant main effects for self- versus experimenter-presentation $[F(1,36)=38.40, p<.01]$ and for the tone

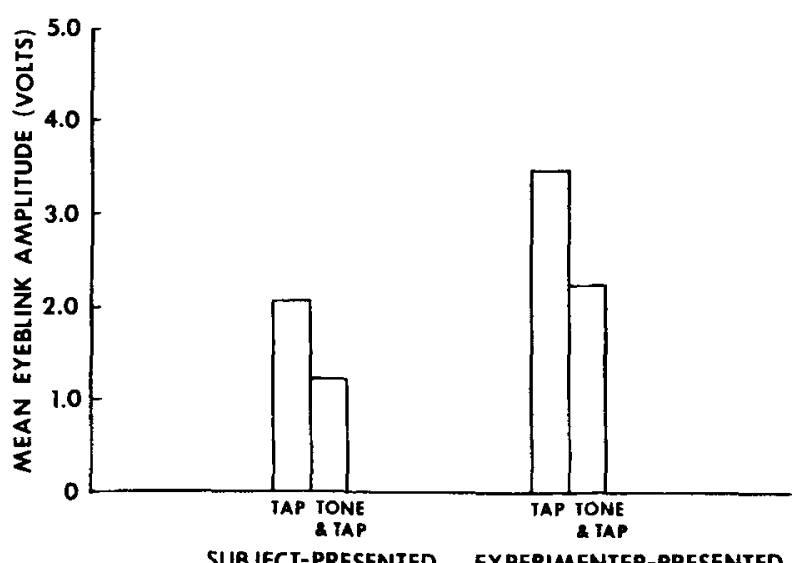

Figure 3. Mean amplitude of elicited eyeblinks when tapa were elther self-presented or presented by the experimenter and when taps were elther preceded by a reflex-inhibiting tone or occurred alone. versus no-tone condition $[F(1,36)=31.01, p<.01]$, with no significant interaction between these two factors.

As shown in Figure 3 and confirmed by the statistical analysis, the difference in amplitude between tapalone and tap-plus-tone conditions was essentially the same on trials when subjects presented their own taps as on trials when the experimenter presented the taps. Moreover, the difference in amplitude between self-presented and experimenter-presented conditions was essentially the same on trials when the tap was preceded by a reflex-modifying tone as when the tap was presented alone. In short, the inhibition engendered by self-presentation and the inhibition engendered by the occurrence of a reflex-modifying tone appears to summate in a simple arithmetic fashion, implying that the two procedures make largely independent contributions to the total amount of inhibition engendered on a given trial (Sternberg, 1969).

\section{EXPERIMENT 4}

Experiments 1 and 2 in the present sequence assessed the inhibitory control exerted by the motor and cognitive factors that are engaged during selfpresentation of a reflex-eliciting stimulus. Experiment 3 sought to determine how these inhibitory factors might combine with the inhibition afforded by an exteroceptive stimulus that leads the reflexeliciting event by an appropriate interval. Although the results of these experiments are obviously relevant to an understanding of the reflex-inhibition phenomenon, none of these experiments was designed to test any particular theoretical account. Experiment 4, on the other hand, was designed to do just this. In particular, it examined the negative perception hypothesis proposed by Lykken and Tellegen (1974). To examine this issue, on some trials subjects received an eyeblink-eliciting tap to the glabella and on others they received an eyeblink-eliciting burst of noise. On a given trial, a subject was either forewarned as to the type of stimulus that would be presented or was denied this information. With this arrangement, one should be able to determine whether or not (as suggested by Lykken \& Tellegen) subjects can "tune" the appropriate receptor system. Moreover, by arranging that on half of the trials the subject would self-present the stimulus while on the rest the experimenter would present the stimulus, it became possible to determine whether, and if so how, the effects of forewarning might interact with the previously described inhibitory effects of self-presentation.

\section{Method}

Subjeets. Seventeen (15 female, 2 male) graduate and undergraduate students from Bryn Mawr College volunteered to participate in this study.

Apparatus. The equipment used for this study was the same as that used in prior experiments, but the program was rearranged so 
that the eyeblink-eliciting stimulus could be either a tap or a noise. As before, the taps were produced by delivery of a $50-\mathrm{msec}, 16-\mathrm{V}$ dc pulse to the tap solenoid. The noise stimulus consisted of $10 \mathrm{msec}$ of $110-\mathrm{dB}$ SPL white noise with a 1 -msec rise-fall time. It was generated by the Coulbourn system described earlier.

Procedure. After a subject had been apprised of the nature of the research and had agreed to participate, he or she was seated in the IAC room and fitted with earphones and the headband holding the tap device and the eyeblink-monitoring device. The subjects were informed that they would be watching a series of colored slides of artworks and nature scenes and that at some interval after each slide change they would receive a stimulus that would elicit an eyeblink. They were then instructed as follows:

"When you press the switch held in your hand you will receive either a tap to your forehead or a loud burst of noise to both ears. The stimulus will occur as soon as you press the switch and is intended to cause you to blink. Do not try to either withhold or augment your blinks and do not press the switch until you are instructed to do so. On some trials you will be told which stimulus (tap or noise) will occur when you press the switch. For example, if you hear 'press tap' over the intercom you will know that you should now press the switch and that you will receive a tap when you do so. On some trials you will be told to press the switch but you will not be told which stimulus to expect. On such trials your verbal instruction will simply be 'press.' On these trials you will receive a tap or a noise when you press the switch, but you will not know which to expect.

"On some trials your verbal instruction will be either 'tap' or 'noise,' but you will not be asked to press the switch. On these trials you will know which stimulus is about to occur, but you will not know exactly when it will occur, as the experimenter, rather than you, will be presenting the stimulus. Finally, on some trials you will be given no instructions. On these trials you will receive either a tap or a noise but you will not know which it will be or when it will occur."

During the session, stimulus presentation occurred at intervals of $20 \pm 5 \mathrm{sec}$. Each subject received one of each kind of trial per block. Overall, there were six blocks of eight trials presented in random order that varied from trial block to trial block.

\section{Results and Discussion}

Experiment 4 was designed to answer the question of whether or not foreknowledge of the type of stimulus to be presented would influence blink amplitude and, if so, whether it enhanced or inhibited the response. Experiment 4 was also designed to reveal whether or not the effect of foreknowledge (whatever its nature) would be independent of the inhibitory effects of self-presentation.

Figure 4 shows the mean amplitude of eyeblink averaged across subjects and across the two types of stimuli. A two-way repeated-measures analysis of variance yielded a significant effect for foreknowledge versus no foreknowledge $[F(1,48)=69.15, p<$ .01] and for self- versus experimenter-presentation $[F(1,48)=17.35, p<.01]$, with no significant interaction between these two factors.

These findings indicate that foreknowledge as to the type of stimulus to be presented inhibits (rather than enhances) the reaction that the stimulus elicits. Moreover, since the effects of foreknowledge entail differentiating between stimulus inputs in different modalities, these findings also imply that the inhibitory effects of foreknowledge occur on the afferent side of the reflex. As such, they support

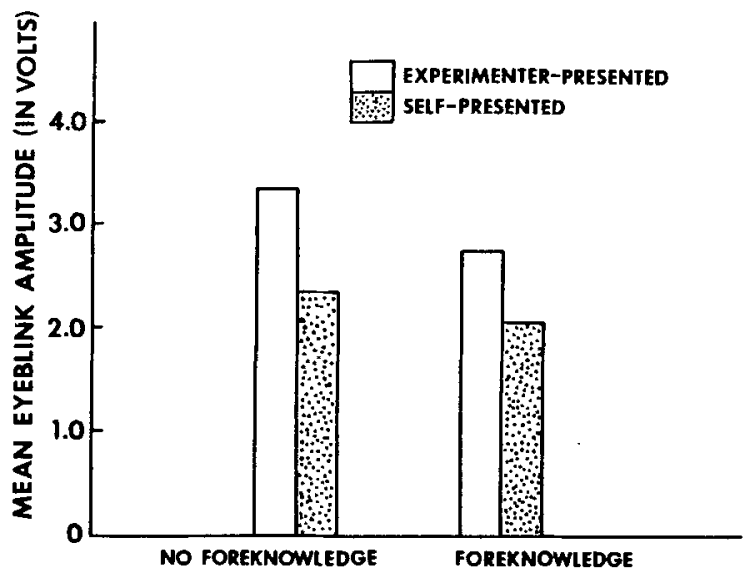

Figure 4. Mean amplitude of elicited eyeblinks averaged acroas stimull (tone and noise) when taps were elther self-presented or presented by the experimenter under conditions in which foreknowledge about the type of eliciting stimulus was either glven or withheld.

Lykken and Tellegen's (1974) negative perception hypothesis, which proposes that subjects can selectively attenuate the appropriate afferent system in anticipation of a stimulus. The findings of Experiment 4 also indicate that the inhibitory effects of foreknowledge as to stimulus type are independent of the reflex inhibition engendered by the motor and cognitive behavior entailed in the act of self-presenting a stimulus.

\section{CONCLUSIONS}

The results of Experiment 1 indicate that when a reflex-eliciting stimulus is self-presented the amplitude of the elicited reaction is reduced. Those results also indicated that the inhibitory process engendered by self-presentation is relatively short lived. It persists for more than $50 \mathrm{msec}$ but less than $500 \mathrm{msec}$ after the overt self-presentation act has occurred.

Experiment 2 examined the inhibitory effects engaged by the motor (or motor-command) components of self-presentation, and it compared them with the inhibitory effects of the subject's expectation at the time that self-presentation occurs. Its results indicate that these two behavioral events make separate and largely independent contributions to the inhibition generated during self-presentation.

Experiment 3 asked how the inhibition afforded by self-presentation might combine with the inhibitory effects that occur when an appropriate reflexmodifying signal precedes a reflex-eliciting stimulus by approximately $100 \mathrm{msec}$. Again, the two kinds of inhibitory processes were found to make largely independent contributions to the total amount of inhibition engendered on a given trial.

Experiment 4 investigated the inhibitory effects engaged when a subject is afforded foreknowledge of 
the nature of a pending reflex-eliciting stimulus. Its results imply that foreknowledge enables a subject to gate input in the appropriate afferent channel.

Considered overall, these findings suggest that the total amount of inhibition that is manifested when a reflex is elicited depends upon the nature and number of inhibitory factors that are engaged at that moment in time. It is of interest that this conclusion is in agreement with that of Ison, Zuckerman, and Russo, (1975). These investigators examined the reflex inhibition engendered by acoustic signals, by visual signals, and by various combinations of these two kinds of signals when they preceded an intense startle-eliciting burst of noise. It was found that when the signals were combined their inhibitory effects added in such a way that a given combination produced more inhibition of the response to the noise burst than either signal alone, but the combined signals did not produce as much inhibition as the arithmetic sum of the effects of the two signals measured separately. In our work, the effects of the several inhibitory factors appear to add in an approximately arithmetic fashion, but it is important to recognize that Ison et al. were examining the inhibitory effects of various combinations of exteroceptive stimuli (e.g., tones and lights), whereas the inhibitory factors studied in the present work entailed the interoceptive stimulation provided by such factors as expectation, foreknowledge, and the motor (or motor command) components of self-presentation.

\section{REFERENCES}

Bjonk8trand, P.A. Electrodermal responses as affected by subject versus experimenter controlled noxious stimulation. Journal of Experimental Psychology, 1973, 97, 365-369.

Dykman, B. M., \& Ison, J. R. Temporal integration of acoustic stimulation obtained in reflex inhibition in rats and humans. Journal of Comparative \& Physiological Psychology, 1979, 93, 939-945.

Furedy, J. J., \& KLajner, F. On evaluating autonomic and verbal indices of negative perception. Psychophysiology, 1974, 11, 121-124.

Graham, F. K., \& Murray, G. M. Discordant effects of weak prestimulation on magnitude and latency of the reflex blink. Physiological Psychology, 1977, 5, 108-114.
Grings, W. W. Preparatory set variables related to classical conditioning of autonomic responses. Psychological Review, $1960,67,243-252$.

HAGGARD, E. Some conditions determining adjustment during and readjustment following experimentally induced stress. Journal of Experimental Psychology, 1943, 33, 257-284.

Hoffman, H. S., Cohen, M. E., \& Stitt, C. L. Acoustic augmentation and inhibition of the human eyeblink. Journal of Experimental Psychology: Human Perception \& Performance, $1981,7,1357-1362$.

Horfman, H. S., \& Ison, J. R. Reflex modification in the domain of startle: 1. Some empirical findings and their implications for how the nervous system processes sensory input. Psychological Review, 1980, 87, 175-189.

Ison, J. R., Zuckerman, M., \& Russo, J. M. Combination rules for inhibitory stimuli. Journal of Experimental Psychology: Animal Behavior Processes, 1975, 1, 318-325.

Krauter, E. E., Leonard, D. W., \& Ison, J. R. Inhibition of the human eyeblink by a brief acoustic stimulus. Journal of Comparative \& Physiological Psychology, 1973, 2, 28-37.

LykKen, D. T., \& Tellegen, A. On the validity of the perception hypothesis. Psychophysiology, 1974, 11, 125-132.

Maltzman, I., \& Wolff, C. Preference for immediate versus delayed noxious stimulation and concomittant GSR. Journal of Experimental Psychology, 1970, 83, 76-79.

MAr9h, R. R., \& Hormman, H. S. Eyeblink elicitation and measurement in the human infant: $A$ circuit modification. Behavior Research Methods \& Instrumentation, 1981, 13, 707.

Marsh, R. R., Hofrman, H. S., \& STITT, C. L. Eyeblink elicitation and measurement in the human infant. Behavior Research Methods \& Instrumentation, 1979, 11, 498-502.

SANEs, J. N. Excitability of cutaneous eyeblink reflex in humans during organization and performance of voluntary movements. Unpublished doctoral dissertation, University of Rochester, 1979.

Silvergtein, L. D., \& Graham, F. K. Obicularis oculi excitability and prestimulation effects during REM and NREM sleep. Psychophysiology, 1979, 16, 177. (Abstract)

Staub, E., Turgxy, B., \& Schwartz, G. E. Self-control and predictability: Their effects on reactions to aversive stimulation. Journal of Personality and Social Psychology, 1971, 18, 157-162.

Sternbera, S. The discovery of processing stages: Extensions of Donder's method. Acta Psychologica, 1969, 30, 276-315.

StitT, C. L., Hormuan, H. S., Mansh, R. R., \& Schwantz, G. M. Modification of the pigeon's visual startle reaction by the sensory environment. Journal of Comparative and Physiological Psychology, 1976, 90, 601-619.

YERKEs, R. M. The sense of hearing in frogs. Journal of Comparative Neurology \& Psychology, 1905, 15, 279-304.

(Manuscript received February 3, 1983; revision accepted for publication April 12, 1983.) 